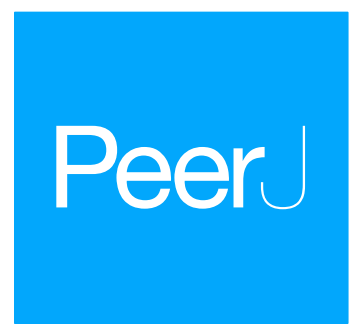

Submitted 4 December 2017 Accepted 11 January 2018 Published 15 February 2018

Corresponding author Juan Nunez-Iglesias, juan.n@unimelb.edu.au, jni.soma@gmail.com

Academic editor Marcelo Ferreira

Additional Information and Declarations can be found on page 7

DOI 10.7717/peerj.4312

Copyright

2018 Nunez-Iglesias et al.

Distributed under

Creative Commons CC-BY 4.0

OPEN ACCESS

\section{A new Python library to analyse skeleton images confirms malaria parasite remodelling of the red blood cell membrane skeleton}

\author{
Juan Nunez-Iglesias ${ }^{1, *}$, Adam J. Blanch ${ }^{2, *}$, Oliver Looker ${ }^{2}$, Matthew W. Dixon ${ }^{2}$ \\ and Leann Tilley ${ }^{2}$ \\ ${ }^{1}$ Melbourne Bioinformatics, The University of Melbourne, Melbourne, Australia \\ ${ }^{2}$ Department of Biochemistry and Molecular Biology, Bio21 Institute, University of Melbourne, Melbourne, \\ Australia \\ These authors contributed equally to this work.
}

\section{ABSTRACT}

We present Skan (Skeleton analysis), a Python library for the analysis of the skeleton structures of objects. It was inspired by the "analyse skeletons" plugin for the Fiji image analysis software, but its extensive Application Programming Interface (API) allows users to examine and manipulate any intermediate data structures produced during the analysis. Further, its use of common Python data structures such as SciPy sparse matrices and pandas data frames opens the results to analysis within the extensive ecosystem of scientific libraries available in Python. We demonstrate the validity of Skan's measurements by comparing its output to the established Analyze Skeletons Fiji plugin, and, with a new scanning electron microscopy (SEM)-based method, we confirm that the malaria parasite Plasmodium falciparum remodels the host red blood cell cytoskeleton, increasing the average distance between spectrin-actin junctions.

Subjects Biochemistry, Bioinformatics, Parasitology, Computational Science Keywords Python, Skeleton analysis, Malaria, Red blood cell, Cytoskeleton

\section{INTRODUCTION}

Skeletons are single-pixel thick representations of networks within an image, and have wide application to understanding the structural properties of objects. For example, skeletons have been used to model human poses, neuronal morphology, nanofibre structure, road networks, kidney development, and vascular networks, among others (Yim, Choyke \& Summers, 2000; Sundar et al., 2003; Bas \& Erdogmus, 2011; Yuan et al., 2009; MoralesNavarrete et al., 2015; Sambaer, Zatloukal \& Kimmer, 2011). These applications include both 2D and 3D images, and often 3D images collected over time, underscoring the need for skeleton analysis software to support multiple imaging modalities and dimensionality.

In this paper, we report Skan, a Python library that produces graphs and branch statistics from skeleton images. Skan is written in Python using the Numba just-in-time (JIT) compiler (Lam, Pitrou \& Seibert, 2015) for performance-critical code, including graph building and graph statistics computation. The source code is available at https://github.com/jni/skan (under a BSD 3-clause license), and we encourage readers 
to contribute code or raise GitHub issues where they require additional functionality to meet their needs. Skan can be installed using standard tools from the two leading Python repositories, the Python Package Index (PyPI) and conda-forge. Installation and usage instructions are available at https://jni.github.io/skan.

Skan works transparently with images of any dimensionality, allowing the analysis of 2D and 3D skeletons. Out of the box, Skan provides functions to compute the pixel skeleton graph, compute statistics about the branches of the skeleton, and draw skeletons and statistical overlays for 2D images.

The pixel skeleton graph maps which pixel is connected to which others in the skeleton image, as well as the distances between them. This graph is provided in the standard scipy.sparse.csr_matrix sparse matrix format, enabling further analysis using common tools for graph and array manipulation in the scientific Python ecosystem.

From this graph, we can compute statistics about the branches of the skeleton, defined as junction-junction and junction-endpoint paths in the pixel skeleton graph. These statistics include average branch length, branch type, branch curvature, branch endpoints, branch euclidean length, and average image intensity along the branch. We return these statistics as a pandas DataFrame, the de-facto standard format for data tables in Python. The table includes the pixel IDs of the branch endpoints, allowing further analysis of the junction-junction graph. Indeed, increasing the breadth of statistics computed by the software was the primary motivation for Skan's development.

Skan further provides a rudimentary GUI to analyse batches of input images. The output of the GUI interface is an Excel file, which contains all the above-mentioned statistics, as well as all analysis parameters, to aid future reproducibility.

Because Skan uses common scientific Python data structures internally, it is easy to extend with new statistics and analyses. The DataFrame of branch statistics follows the "tidy data" paradigm (Wickham, 2014), with each row representing one branch of a skeleton, facilitating downstream analysis, such as computing summary statistics for each disjoint skeleton in an image.

To demonstrate Skan's 3D capabilities, we first compared its output to that of Fiji's Analyze Skeletons (Arganda-Carreras et al., 2010), applied to a publicly available dataset of neuron skeleton traces. Then, we used Skan to measure the spectrin cytoskeleton on the cytoplasmic side of the plasma membrane of red blood cells (RBCs) infected with the malaria parasite Plasmodium falciparum, using a new SEM-based protocol, and confirmed the remodelling of the RBC membrane skeleton by the parasite.

\section{METHODS}

\section{Analysis of skeleton model from DIADEM challenge}

We downloaded the olfactory projection neuron 1 (OP-1) model as a SWC file from DIADEM's website at http://diademchallenge.org/data_set_downloads.html, along with its corresponding 3D TIFF image stack. We then rasterised the model (i.e., converted it from a network of vertex coordinates to a set of active pixels) by using the Simple Neurite Tracer (Longair, Baker \& Armstrong, 2011) plugin for Fiji, function "Analysis > 
A
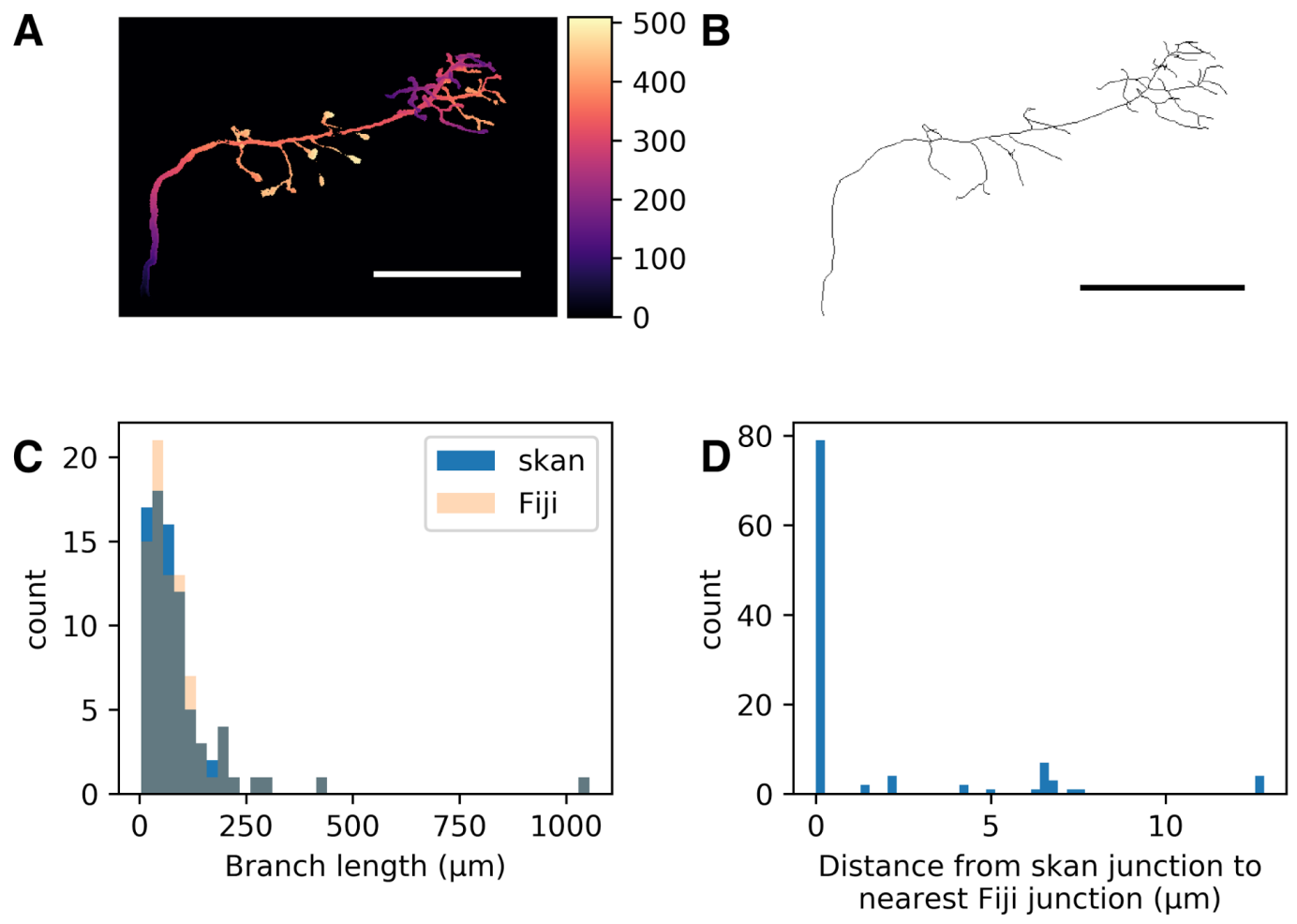

Figure 1 Comparison of skan and Fiji analysis results of the neuronal skeleton from olfactory projection neuron 1 (OP-1) from the DIADEM challenge. (A) Depth projection of the neuron. Scale bar: $500 \mu \mathrm{m}$. Colour map: height in $\mu \mathrm{m}$. (B) Skeleton of the neuron. (C) Distribution of 82 branch lengths between 103 branch points measured by Skan and Fiji in the neuronal skeleton. (D) Distance from 103 skan junction points to the nearest Fiji junction point. Note that the voxel spacing is approximately $9 \times 3 \times$ $3 \mu \mathrm{m}$, so almost all of these distances are less than one pixel apart.

Full-size DOI: 10.7717/peerj.4312/fig-1

Render/Analyze Skeletonized Paths." This produces a 6-connected skeleton path, which we needed to convert to a (thinner) 26-connected path, so we further skeletonized the raster with the morphology . skeletonize3d function from scikit-image (Van der Walt et al., 2014), and saved it as a compressed TIFF file.

Then, we imported this raster image into either Fiji or Python (using Christoph Gohlke's TIFF file). In both cases, we manually set the scale to $9.100602 \times 3.033534 \times 3.033534 \mu \mathrm{m}$ per voxel, as documented on the DIADEM website. In Fiji, we used "Analyze Skeletons" with the "Show detailed info" option ticked, saved the results to csv, and loaded them into a pandas DataFrame in Python. In Python, we used skan.csr. summarise to produce a corresponding pandas DataFrame for Skan's analysis. Finally, we used numpy. histogram and matplotlib.pyplot.hist (Hunter, 2007) to produce the histogram in Fig. 1.

\section{Tissue origin and ethics approval}

This study made use of donated human red blood cells. All experiments were approved by The University of Melbourne School of Biomedical Sciences, Human Ethics Advisory Group (HEAG), for project titled "Characterising host cell interactions in the human malaria parasite, Plasmodium falciparum”, and ethics ID 1135799. Cells were obtained 
by a Material Supply Agreement with the Australian Red Cross Blood Service, agreement number-17-05VIC-23.

\section{Sample preparation and SEM imaging}

To prepare sheared membranes, infected and uninfected red blood cells were attached to 3-Aminopropyl-triethoxysilane treated glass slides using the lectin erythro-agglutinating phytohemagglutinin (PHA-E) and sheared in a hypotonic buffer according to a previously established procedure (Shi et al., 2013). Sheared membranes were immediately fixed with $2.5 \%$ glutaraldehyde for $1 \mathrm{~h}$ before dehydration in a series of ethanol:water mixtures of 20 , $50,70,80,90,95$ and $(3 \times) 100 \%$ ethanol for 5 min each and finally being allowed to dry in air.

Dried samples were gold coated on the rotating mount of a Dynavac SC100 sputter coating instrument for $35 \mathrm{~s}$ using a $25 \mathrm{~mA}$ current, measuring $0.2 \mathrm{~nm}$ thickness on the quartz crystal microbalance. The coating procedure was optimised to prevent under- or overcoating which presents problems with the skeleton trace.

SEM images were recorded using the ETD detector (in Optiplan mode) of an FEI Teneo instrument with a working distance of $5 \mathrm{~mm}$, a beam current of $50 \mathrm{pA}$ and a $2 \mathrm{kV}$ accelerating voltage. Multiple images at 200-250 k magnification were recorded per individual cell to cover a greater portion of the membrane.

\section{Extraction of skeleton data from SEM images}

In our SEM images, the spectrin-actin network appears as bright (raised) patches over dark patches of background (see Fig. 1). We followed a simple approach to trace the midline of the spectrin branches: smoothing the images, then thresholding them (Sauvola \& Pietikäinen, 2000), and finally thinning them (Zhang \& Suen, 1984). The width of the Gaussian smoothing, the window size for thresholding, and the offset for the thresholding are all parameters of our approach, and are recorded in the results output file of a skeleton analysis (when using the graphical user interface).

\section{Data and code availability}

Our code is open source and available at https://github.com/jni/skan. Its documentation can be viewed at https://jni.github.io/skan and includes all code necessary to reproduce Fig. 2. Additional scripts used in our analyses are available at https://github.com/jni/skan-scripts.

We have made the schizont SEM dataset available at the Open Science Framework (OSF), with DOI 10.17605/OSF.IO/SVPFU, together with an archive of the documentation at time of publication, and a sample Excel file resulting from analysing the schizont dataset using the GUI.

\section{RESULTS}

\section{Comparison to Fiji's analyze skeletons plugin}

As a check that our software was producing results consistent with the existing literature on skeleton analysis, we compared our software's results with that of Fiji's Analyze Skeletons plugin (Arganda-Carreras et al., 2010). Although the original data from that 
A
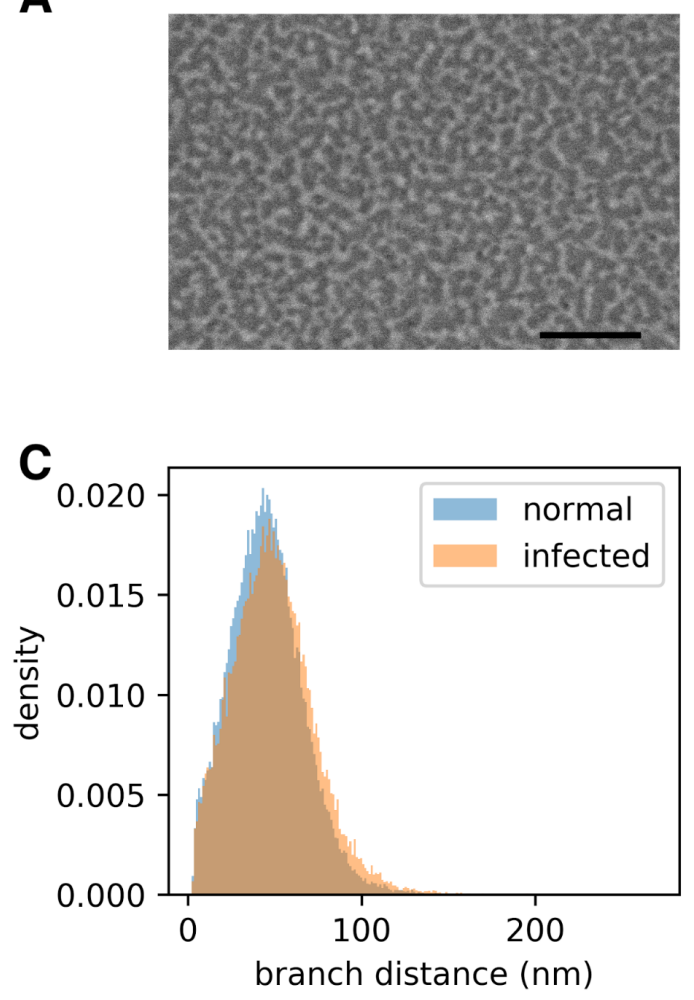

B
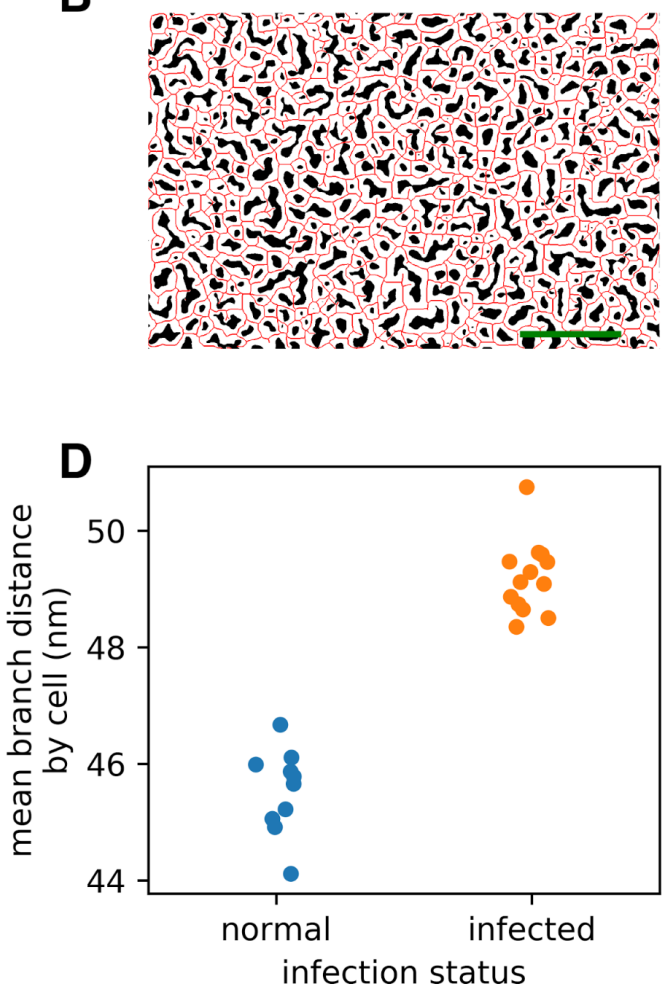

Figure 2 Infection by the malaria parasite remodels the spectrin skeleton of the host red blood cell in the asexual developmental stage. (A) Example image produced by our protocol. Scale bar: $300 \mathrm{~nm}$. (B) Thresholding (white) and skeletonisation (red) of the image in (A). (C) Complete distribution of measured spectrin branch lengths for normal and infected RBCs. (D) Mean spectrin branch length by cell $\left(n_{\text {norm }}=10, n_{\text {inf }}=13\right)$.

paper is unavailable (I Arganda-Carreras, pers. comm., 2017), we compared the output of our software with that of Analyze Skeletons on a neuron skeleton from the DIADEM Challenge (http://diademchallenge.org) (Figs. 1A-1B). Both software packages found the same number of skeleton branches, with very close agreement between the two branch length distributions (Fig. 1C) and branch point locations (Fig. 1D). Manual inspection confirmed that the small differences observed result from the different treatment of branch junctions (see Supplementary information).

\section{Malaria parasites remodel the red blood cell inner membrane cytoskeleton}

Prior studies have shown that infection by $P$. falciparum, the most deadly malaria-causing parasite, results in changes in the physical properties of the infected red blood cell (iRBC), and that these changes are associated with an elongation of the spectrin skeleton branches in the inner RBC membrane skeleton (Shi et al., 2013; Dearnley et al., 2016; Nans, Mohandas $\&$ Stokes, 2011). A coarse-grained molecular model suggested that this spectrin stretching could, in part, account for the deformability changes of the iRBC (Dearnley et al., 2016), emphasizing the biological significance of the measurements. We sought to confirm 
these observations using a novel scanning electron microscopy (SEM)-based protocol (A Blanch et al., 2017, unpublished data). The method involves cross-linking the RBCs to a glass coverslip and shearing off the upper membrane component, thus exposing the cytoplasmic/internal side of the cross-linked plasma membrane (Shi et al., 2013). The membrane is chemically fixed, dehydrated and gold-coated before imaging (Fig. 2A). We automatically extracted spectrin skeletons (Fig. 2B) from images produced using both uninfected RBCs and RBCs infected with mature stage parasites (40-44 h post infection). We found that the average spectrin branch distance increased from $45.5 \mathrm{~nm}$ to $49.2 \mathrm{~nm}$, an increase of $8 \%$ (Figs. 2C-2D).

\section{DISCUSSION AND CONCLUSIONS}

\section{Spectrin remodelling by $P$. falciparum}

The remarkable deformability and durability of the RBC membrane derives from its membrane skeleton (Zhang et al., 2015). The skeleton is composed of a regular hexagonal array of "spring-like" proteins forming a meshwork at the cytoplasmic surface of the RBC. Spectrin heterodimers constitute the cross-beams of the molecular architecture and are connected to integral membrane proteins in the plasma membrane. Previous studies using atomic force microscopy (AFM) and transmission electron microscopy (TEM), followed by manual selection and measurement of skeleton branches, revealed reorganization and expansion of the spectrin network of the host cell membrane (Shi et al., 2013; Millholland et al., 2011; Cyrklaff et al., 2011).

In this work we have applied a novel SEM-based method to image the RBC membrane skeleton, and a fully automated method for selection and measurement of the spectrin branch distances. We observed an $8 \%$ increase in the length of the spectrin cross-members, in reasonable agreement with previous studies. Our data are consistent with the Cyrklaffet al. (2011) cryo-electron tomography study that provided evidence that the RBC membrane skeleton is reorganised as a result of mining of the actin junction points to generate actin filaments that connect parasite-derived organelles known as Maurer's clefts to the knobs.

\section{Numba and performance}

An interesting aspect of Skan's development is its use of Numba, a just-in-time compiler for Python code. Skan is one of the first scientific packages to make extensive use of Numba to speed up its operations. In our hands, Numba has been able to dramatically speed up our code, in some cases approaching the theoretical maximum performance of our CPUs.

As just one example, in the context of implementing Sauvola image thresholding, we developed a function for the cross-correlation of an $n$-dimensional image with a sparse kernel. Sauvola thresholding requires computing the local mean and standard deviation for every pixel in an image. This can be optimally achieved by computing the integral of both the original image and the image of squared intensity values, and then convolving each with a kernel consisting of the outer product of the vector $(-1,0,0, \ldots, 0,1)$ with itself, where the number of zeros is equal to the width of the neighbourhood minus one. This definition results in an extremely sparse kernel, which is not efficiently used by 
conventional convolution functions available in NumPy (v1.12) and SciPy (v0.19) (Van der Walt, Colbert \& Varoquaux, 2011; Oliphant, 2007).

The function is implemented as correlate_sparse, which handles boundary conditions and formatting of the kernel, and then calls the Numba-jitted function _correlate_sparse_offsets, which iterates through the array, performing the crosscorrelation.

The result is striking. For a 2,048 by 2,048 pixel image and a 31 by 31 kernel size, correlate_sparse takes $130 \mathrm{~ms}$, somewhat slower than SciPy's ndimage.correlate, which takes 57 ms. For a much bigger 301 by 301 kernel, however, correlate_sparse takes a similar amount of time- $135 \mathrm{~ms}$ - while SciPy takes $17 \mathrm{~s}$. Furthermore, if we analyse just the inner loop of the computation, the part handled by Numba, we measured a time of $1.8 \mathrm{~ns}$ per loop in our $1.3 \mathrm{GHz}$ (i.e., $0.77 \mathrm{~ns}$ per cycle) CPU. Each loop performs two additions and a multiplication, in addition to array access, suggesting that Numba is close to achieving optimal performance for our problem and CPU. This example illustrates the power of Numba to speed up numerical Python code.

We also take this opportunity to note the loop order in the code of _correlate_sparse_ offsets. For every non-zero element of the kernel, we make a full pass over the input image. When picturing a convolution, this is slightly counter-intuitive: most people would instead consider, for each pixel position, correlating all the non-zero elements of the kernel (thus examining each pixel only once).

However, that order of operations is poorly optimised for modern processor architectures, which fetch RAM contents by chunks into the processor cache. Once a chunk has been loaded, accessing elements of that chunk is 20-200 times faster than fetching more data from RAM (Jonas Bonér, https://gist.github.com/jboner/2841832). One consequence is that algorithms that access data in the order in which it is stored in RAM end up being much faster, by virtue of using processor cache to the maximum extent possible.

In our case, this translated to a 10-fold speedup when changing the order from (for pixel in image: for elem in kernel) to (for elem in kernel: for pixel in image), even though these two expressions are mathematically equivalent.

\section{ACKNOWLEDGEMENTS}

We thank Ignacio Arganda-Carreras for suggesting the dataset to compare Skan to Analyze Skeletons, and Alan Rubin for help with Skan's GUI code. We also thank Matthew Brett for the nb2plots library, which we used to write the documentation for the software.

\section{ADDITIONAL INFORMATION AND DECLARATIONS}

\section{Funding}

This work was supported by the Australian Research Council (grant number FL150100106). The funders had no role in study design, data collection and analysis, decision to publish, or preparation of the manuscript. 


\section{Grant Disclosures}

The following grant information was disclosed by the authors:

Australian Research Council: FL150100106.

\section{Competing Interests}

The authors declare there are no competing interests.

\section{Author Contributions}

- Juan Nunez-Iglesias conceived and designed the experiments, analyzed the data, contributed reagents/materials/analysis tools, wrote the paper, prepared figures and/or tables.

- Adam J. Blanch conceived and designed the experiments, performed the experiments, analyzed the data, contributed reagents/materials/analysis tools, wrote the paper, reviewed drafts of the paper.

- Oliver Looker and Matthew W. Dixon performed the experiments, contributed reagents/materials/analysis tools.

- Leann Tilley conceived and designed the experiments, wrote the paper, reviewed drafts of the paper.

\section{Human Ethics}

The following information was supplied relating to ethical approvals (i.e., approving body and any reference numbers):

The University of Melbourne, School of Biomedical Sciences, Human Ethics Advisory Group (HEAG) provided ethical approval for this study (ETHICS ID -1135799).

\section{Data Availability}

The following information was supplied regarding data availability:

Code: https://github.com/jni/skan.

Data: Open Science Framework public project, DOI 10.17605/OSF.IO/SVPFU. https://osf.io/svpfu/.

\section{Supplemental Information}

Supplemental information for this article can be found online at http://dx.doi.org/10.7717/ peerj.4312\#supplemental-information.

\section{REFERENCES}

Arganda-Carreras I, Fernández-González R, Muñoz-Barrutia A, Ortiz-De-Solorzano C. 2010. 3D reconstruction of histological sections: application to mammary gland tissue. Microscopy Research and Technique 73(11):1019-1029 DOI 10.1002/jemt.20829.

Bas E, Erdogmus D. 2011. Principal curves as skeletons of tubular objects. Neuroinformatics 9(2-3):181-191 DOI 10.1007/s12021-011-9105-2.

Cyrklaff M, Sanchez CP, Kilian N, Bisseye C, Simpore J, Frischknecht F, Lanzer

M. 2011. Hemoglobins $S$ and $C$ interfere with actin remodeling in plasmodium 
falciparum-infected erythrocytes. Science 334(6060):1283-1286

DOI 10.1126/science.1213775.

Dearnley M, Chu T, Zhang Y, Looker O, Huang C, Klonis N, Yeoman J, Kenny S, Arora M, Osborne JM, Chandramohanadas R, Zhang S, Dixon MWA, Tilley L. 2016.

Reversible host cell remodeling underpins deformability changes in malaria parasite sexual blood stages. Proceedings of the National Academy of Sciences of the United States of America 113(17):4800-4805 DOI 10.1073/pnas.1520194113.

Hunter JD. 2007. Matplotlib: a 2D graphics environment. Computing in Science \& Engineering 9(3):90-95 DOI 10.1109/MCSE.2007.55.

Lam SK, Pitrou A, Seibert S. 2015. Numba: a LLVM-based python JIT compiler. In: Proceedings of the second workshop on the LLVM compiler infrastructure in HPC, LLVM '15. New York: ACM, 7:1-7:6.

Longair MH, Baker DA, Armstrong JD. 2011. Simple Neurite Tracer: open source software for reconstruction, visualization and analysis of neuronal processes. Bioinformatics 27(17):2453-2454 DOI 10.1093/bioinformatics/btr390.

Millholland MG, Chandramohanadas R, Pizzarro A, Wehr A, Shi H, Darling C, Lim CT, Greenbaum DC. 2011. The malaria parasite progressively dismantles the host erythrocyte cytoskeleton for efficient egress. Molecular \& Cellular Proteomics 10(12):M111.010678 DOI 10.1074/mcp.M111.010678.

Morales-Navarrete H, Segovia-Miranda F, Klukowski P, Meyer K, Nonaka H, Marsico G, Chernykh M, Kalaidzidis A, Zerial M, Kalaidzidis Y. 2015. A versatile pipeline for the multi-scale digital reconstruction and quantitative analysis of $3 \mathrm{D}$ tissue architecture. eLife 4:e11214 DOI 10.7554/eLife.11214.

Nans A, Mohandas N, Stokes DL. 2011. Native ultrastructure of the red cell cytoskeleton by cryo-electron tomography. Biophysical Journal 101(10):2341-2350 DOI 10.1016/j.bpj.2011.09.050.

Oliphant TE. 2007. SciPy: Open source scientific tools for Python. Computing in Science and Engineering 9:10-20.

Sambaer W, Zatloukal M, Kimmer D. 2011. 3D modeling of filtration process via polyurethane nanofiber based nonwoven filters prepared by electrospinning process. Chemical Engineering Science 66(4):613-623 DOI 10.1016/j.ces.2010.10.035.

Sauvola J, Pietikäinen M. 2000. Adaptive document image binarization. Pattern Recognition 33(2):225-236 DOI 10.1016/S0031-3203(99)00055-2.

Shi H, Liu Z, Li A, Yin J, Chong AGL, Tan K. SW, Zhang Y, Lim CT. 2013. Life cycledependent cytoskeletal modifications in Plasmodium falciparum infected erythrocytes. PLOS ONE 8(4):e61170 DOI 10.1371/journal.pone.0061170.

Sundar H, Silver D, Gagvani N, Dickinson S. 2003. Skeleton based shape matching and retrieval. In: Shape modeling international, 2003. 130-139.

Van der Walt S, Colbert SC, Varoquaux G. 2011. The NumPy array: a structure for efficient numerical computation. Computing in Science \& Engineering 13(2):22-30 DOI 10.1109/MCSE.2011.37. 


\section{Van der Walt S, Schönberger JL, Nunez-Iglesias J, Boulogne F, Warner JD, Yager N,}

Gouillart E, Yu T, Scikit-image contributors. 2014. Scikit-image: image processing in Python. PeerJ 2:e453 DOI 10.7717/peerj.453.

Wickham H. 2014. Tidy data. Journal of Statistical Software 59(10):1-23 DOI 10.18637/jss.v059.i10.

Yim PJ, Choyke PL, Summers RM. 2000. Gray-scale skeletonization of small vessels in magnetic resonance angiography. IEEE Transactions on Medical Imaging 19(6):568-576 DOI 10.1109/42.870662.

Yuan X, Trachtenberg JT, Potter SM, Roysam B. 2009. MDL constrained 3-D grayscale skeletonization algorithm for automated extraction of dendrites and spines from fluorescence confocal images. Neuroinformatics 7(4):213-232 DOI 10.1007/s12021-009-9057-y.

Zhang Y, Huang C, Kim S, Golkaram M, Dixon MWA, Tilley L, Li J, Zhang S, Suresh S. 2015. Multiple stiffening effects of nanoscale knobs on human red blood cells infected with Plasmodium falciparummalaria parasite. Proceedings of the National Academy of Sciences of the United States of America 112(19):6068-6073 DOI 10.1073/pnas.1505584112.

Zhang TY, Suen CY. 1984. A fast parallel algorithm for thinning digital patterns. Communications of the ACM 27(3):236-239 DOI 10.1145/357994.358023. 